\title{
Mechanism of reduction of an aminyl radical intermediate in the radical SAM GTP 3',8-cyclase MoaA
}

\author{
Haoran Pang ${ }^{1 \#}$, Lindsey M. Walker ${ }^{2 \#}$, Alexey Silakov#, Pan Zhang ${ }^{4}$, Weitao Yang ${ }^{4}$, Sean J. Elliott ${ }^{2, *}$, \\ and Kenichi Yokoyama ${ }^{1,4, *}$
}

${ }^{1}$ Department of Biochemistry, Duke University School of Medicine, Durham, North Carolina 27710, USA.

${ }^{2}$ Department of Chemistry, Boston University, Boston, Massachusetts 02215, USA.

${ }^{3}$ Department of Chemistry, The Pennsylvania State University, University Park, Pennsylvania 16802, USA.

${ }^{4}$ Department of Chemistry, Duke University, Durham, North Carolina 27710, USA.

*Corresponding Author: ken.yoko@duke.edu, elliott@bu.edu.

\# These authors contributed equally. 


\section{Table of Contents}

Table S1. Reduction potentials of wt- and $\triangle R S-M o a A$ in the presence of SAM and/or GTP measured by square wave voltammograms.

Table S2. GTP and SAM binding effects with wt- or $\triangle R S-M o a A$ determined by anaerobic ITC. ${ }^{a}$ 4

Figure S1. Electrochemical characterization of wt- and $\triangle R S-M o a A$. 5

Figure S2. Pourbaix diagram of wt-MoaA. 6

Figure S3. Square wave voltammograms of wt- and $\triangle R S-M o a A$ in the presence of SAM or GTP. 7

Figure S4. ITC analysis of wt- and $\triangle R S-M o a A$ titrated with SAM or GTP.___ 8

Figure S5. Q-band EPR spectrum of $5^{\prime}$-dA-C4' · along with the [4Fe-4S] signal.__ 9

Figure S6. Structures of different states in DFT calculation of aminyl radical reduction.

Reference 10 11 
Table S1. Reduction potentials of wt- and $\triangle$ RS-MoaA in the presence of SAM and/or GTP measured by square wave voltammograms.

\begin{tabular}{ccc}
\hline & wt-MoaA (mV vs SHE) & $\Delta$ RS-MoaA (mV vs SHE) \\
\hline no substrate & -468 & -450 \\
+ SAM & -464 & -455 \\
+ GTP & -483 & -440 \\
+ SAM + GTP & -480 & -430 \\
\hline
\end{tabular}


Table S2. GTP and SAM binding effects with wt- or $\triangle$ RS-MoaA determined by anaerobic ITC.

\begin{tabular}{ccccc}
\hline enzyme & substrate & \#N (sites) & $K_{d}(\mu \mathrm{M})$ & $\Delta \mathrm{H}(\mathrm{kcal} / \mathrm{mol})$ \\
\hline \multirow{2}{*}{ wt-MoaA } & SAM & $0.91 \pm 0.05$ & $2.42 \pm 0.82$ & $-7.35 \pm 0.53$ \\
\hline GTP & SAM & $0.60 \pm 0.04$ & $3.54 \pm 0.81$ & $-19.9 \pm 2.0$ \\
\hline GTP & $/$ & $>90^{\mathrm{b}}$ & $-15.2 \pm 1.5$
\end{tabular}

a The ITC data was fitted to a one-set of binding sites model by Origin $7 .{ }^{b} K_{d}$ value was above the upper limit of detection for ITC analysis $(90 \mu \mathrm{M})$. 

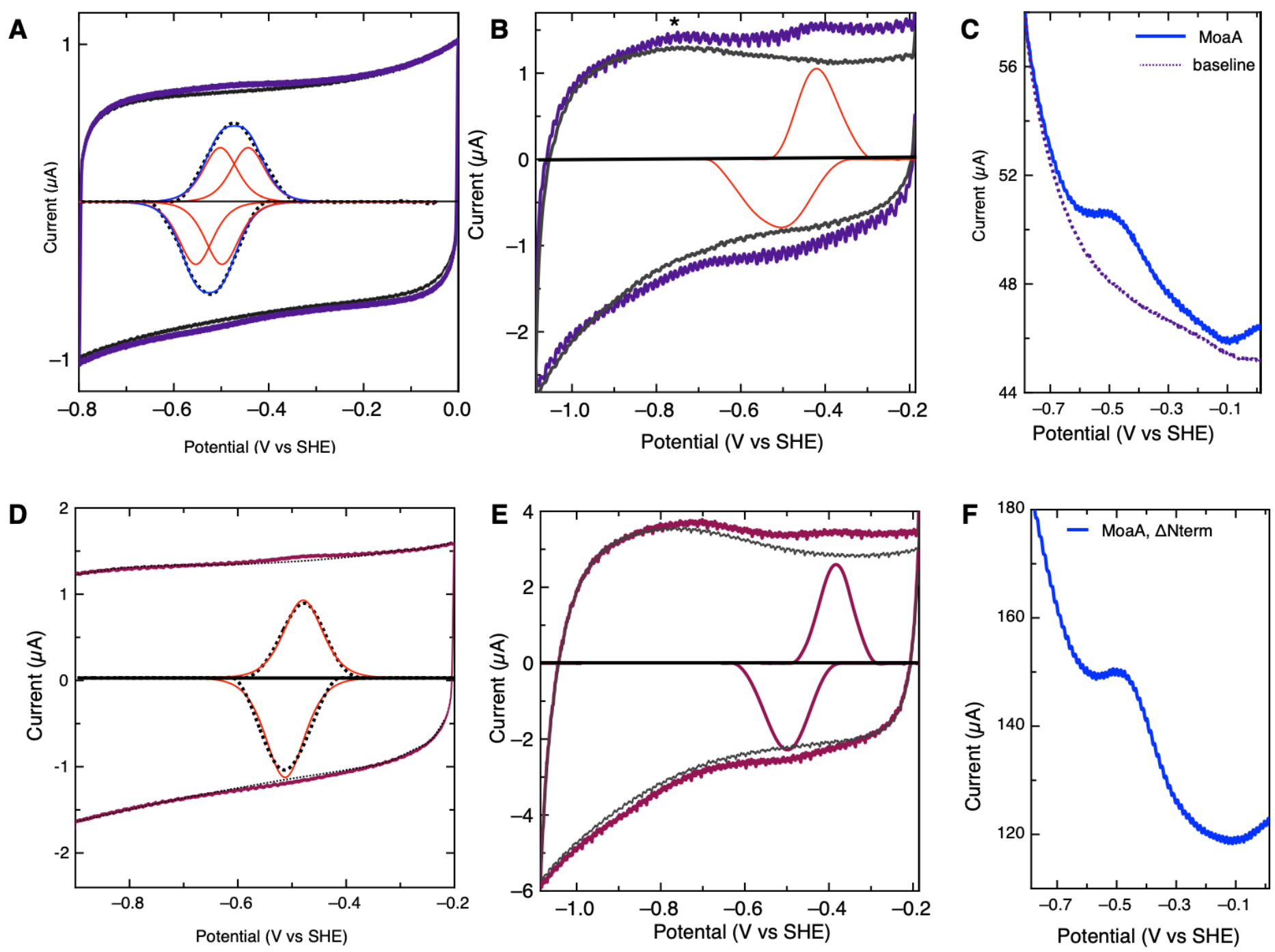

Figure S1. Electrochemical characterization of wt- and $\triangle \mathrm{RS}-\mathrm{MoaA}$.

All measurements were performed at $4{ }^{\circ} \mathrm{C}$ and $\mathrm{pH}$ 7.5. (A) Cyclic voltammogram of wt-MoaA on a pyrolytic graphite edge (PGE) electrode without, and (B) with multi-walled carbon nanotubes (MWCNT) with a scan rate of $200 \mathrm{mV} / \mathrm{s}$ with baseline (grey dot) and protein film (purple). Data that have had non-faradaic component of the current are shown as an inset on a x 20 scale. For wt-MoaA, data were deconvoluted into two features where $n$ =1. The measurement with MWCNT-modified PGE electrode led to an increase in the separation of oxidative and reductive currents, suggesting heterogeneity of conformations of the enzyme at the electrode, and/or a fundamentally slow electron transfer rate. (C) Square wave voltammogram of wt-MoaA measured with a frequency of $15 \mathrm{~Hz}$ and $50 \mathrm{mV}$ amplitude. (D) Cyclic voltammogram of $\triangle R S-M o a A$ on a PGE electrode modified without, and (E) with MWCNT with a scan rate of $100 \mathrm{mV} / \mathrm{s}$ with baseline (grey dot) and protein film (maroon). For $\triangle \mathrm{RS}-\mathrm{MoaA}$ CV data on PGE data were fit well to a single $n=1$ feature, shown as an inset on a $\times 20$ scale (F) Square wave voltammogram of $\Delta R S-M o a A$ measured with a frequency of $15 \mathrm{~Hz}$ and amplitude of $50 \mathrm{mV}$. 


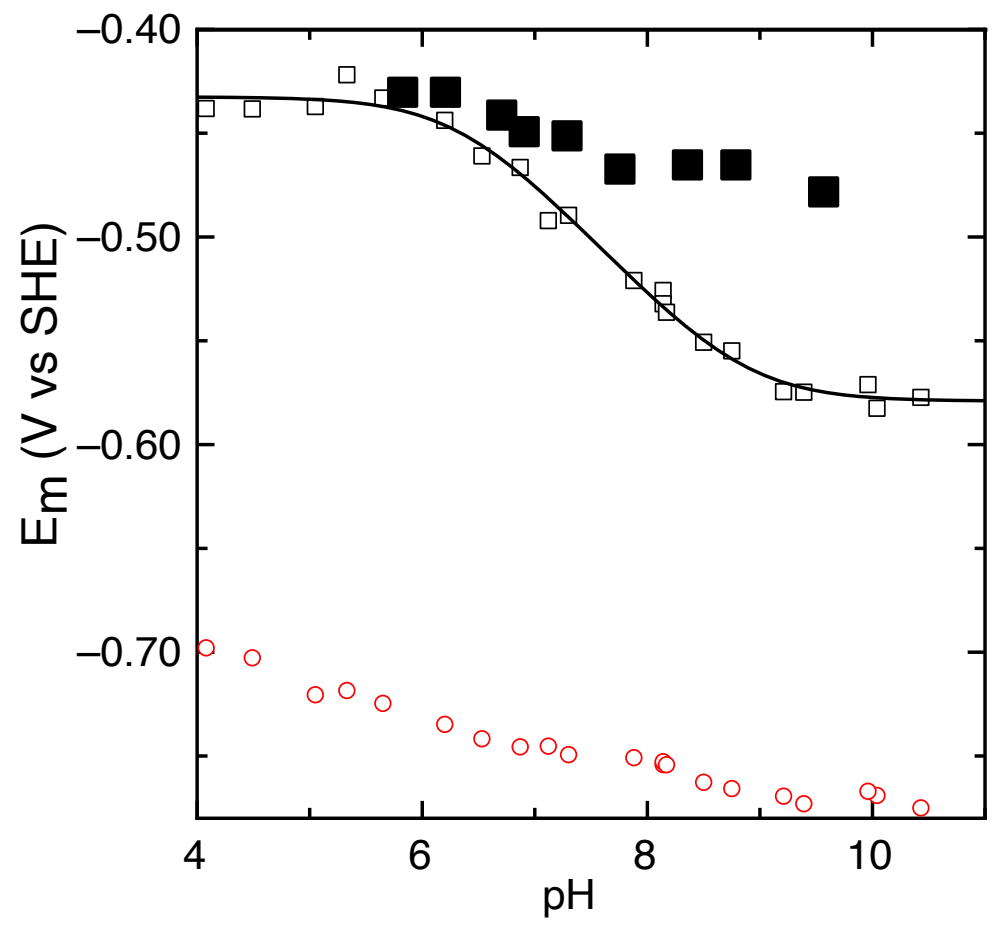

Figure S2. Pourbaix diagram of wt-MoaA.

The $\mathrm{pH}$ dependence of the overall envelop of signals of wt-MoaA, as determined for the enzyme adsorbed at a MWCNT-modified PGE electrode, via SWV at $4{ }^{\circ} \mathrm{C}$, frequency of $15 \mathrm{~Hz}$, and amplitude of $50 \mathrm{mV}$ in multicomponent buffer ranging in $\mathrm{pH}$ from 5.5 to 9.5 (solid squares). For the sake of comparison, analogous data is reported for the radical SAM enzyme BtrN ${ }^{1}\left([4 \mathrm{Fe}-4 \mathrm{~S}]_{\mathrm{RS}}\right.$, open squares; $[4 \mathrm{Fe}-4 \mathrm{~S}]_{A U X}$, open circles), which has a His117 residue within an $\mathrm{H}$-bonding distance from the $[4 \mathrm{Fe}-4 \mathrm{~S}]_{R S}$ cluster. For a one proton coupled to one electron process, a slope of $-56 \mathrm{mV}$ per pH unit is expected at $4{ }^{\circ} \mathrm{C}$, while only about $-12 \mathrm{mV}$ change per $\mathrm{pH}$ unit was observed indicating no $\mathrm{pH}$-dependent shift in the reduction potential. 

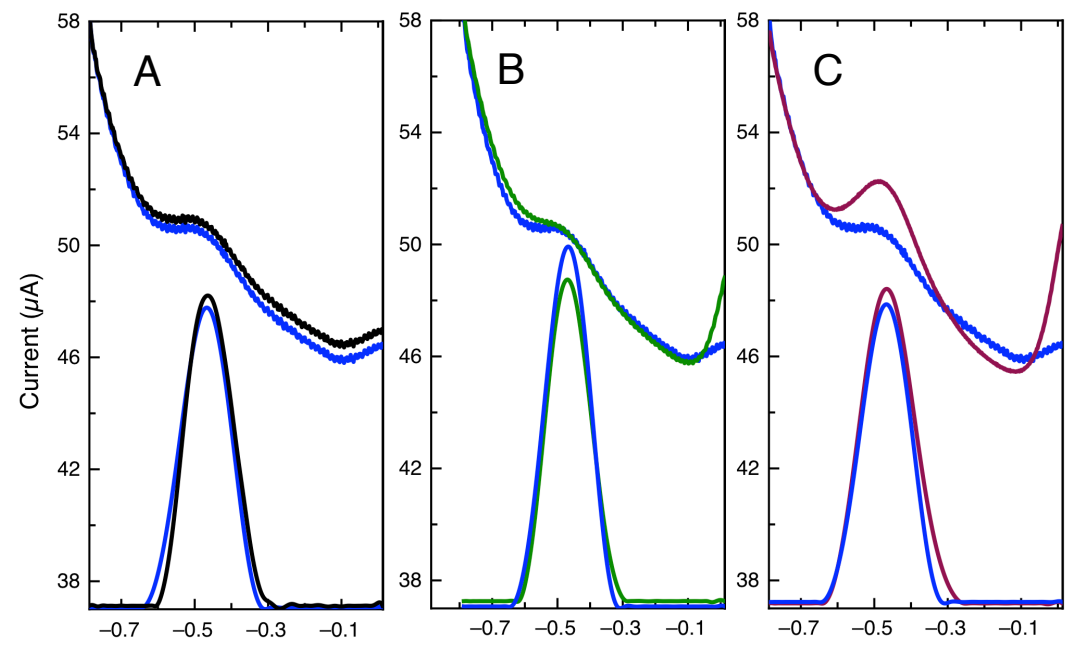

Potential (V vs SHE)

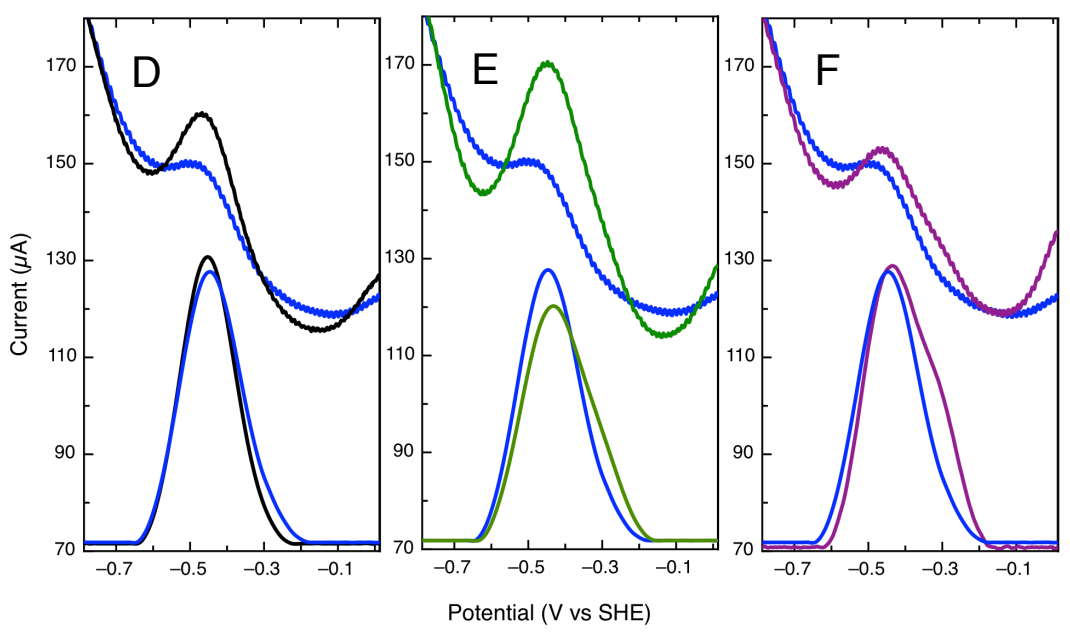

Figure S3. Square wave voltammograms of wt- and $\triangle$ RS-MoaA in the presence of SAM or GTP.

Square wave voltammetry of wt-MoaA (300 $\mu \mathrm{M}$, blue) in the presence of $1 \mathrm{mM}$ SAM (black) (A), $1 \mathrm{mM}$ GTP (green) (B), or $1 \mathrm{mM} \mathrm{SAM}$ and $1 \mathrm{mM} \mathrm{GTP}$ (maroon) (C) measured at $4{ }^{\circ} \mathrm{C}, \mathrm{pH} 7.5$, amplitude of $50 \mathrm{mV}$ and frequency of $10 \mathrm{~Hz}$. Square wave voltammetry of $\triangle \mathrm{RS}-\mathrm{MoaA}(300 \mu \mathrm{M}$, blue) in the presence of $1 \mathrm{mM}$ SAM (black) (D), 1 mM GTP (green) (E), or $1 \mathrm{mM} \mathrm{SAM}$ and $1 \mathrm{mM} \mathrm{GTP} \mathrm{(violet)} \mathrm{(F)} \mathrm{measured} \mathrm{at} 4{ }^{\circ} \mathrm{C}, \mathrm{pH} 7.5$, amplitude of $50 \mathrm{mV}$ and frequency of $15 \mathrm{~Hz}$. 

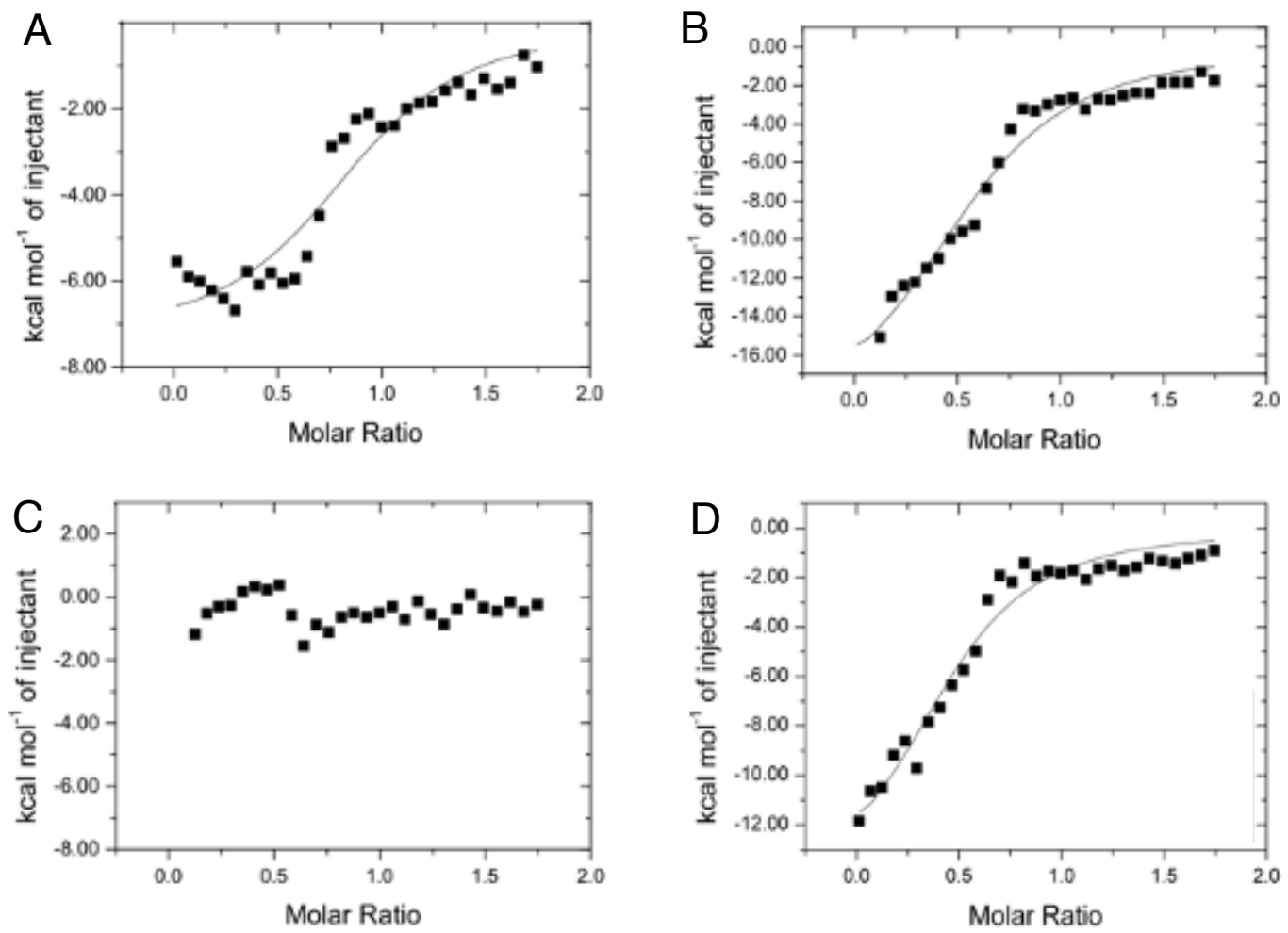

Figure S4. ITC analysis of wt- and $\triangle$ RS-MoaA titrated with SAM or GTP.

$1.5 \mathrm{~mL}$ of $20 \mu \mathrm{M}$ enzyme was titrated with $200 \mathrm{uM}$ of SAM or GTP. (A) wt-MoaA titrated with SAM, (B) wt-MoaA titrated with GTP, (C) $\triangle$ RS-MoaA titrated with SAM, (D) $\triangle$ RS-MoaA titrated with GTP. The ITC data was fitted to the single set of identical binding sites model by Origin 7 and MicroCal ITC Analysis Software (Malvern). 


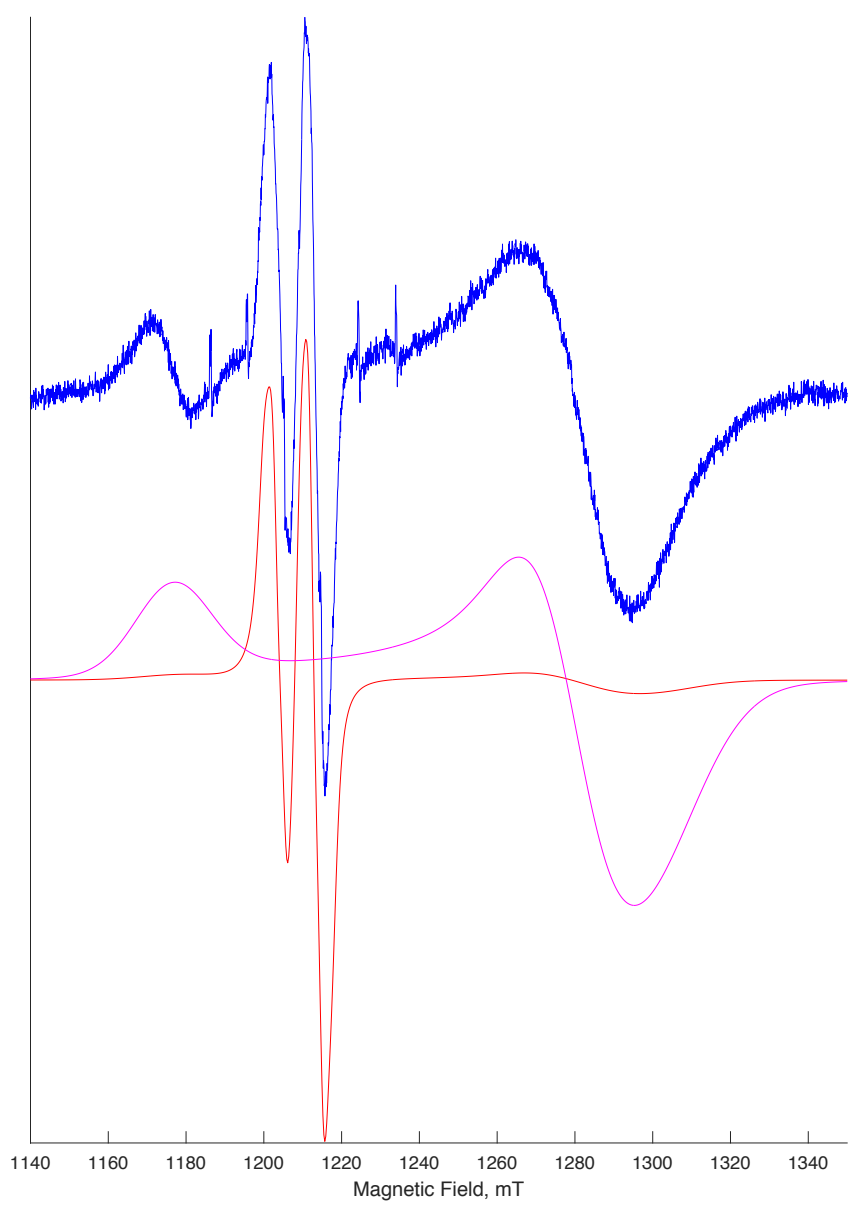

Figure S5. Q-band EPR spectrum of 5'-dA-C4'• along with the [4Fe-4S] signal.

Q-band EPR spectrum of MoaA sample with non-labeled SAM and GTP measured at $10 \mathrm{~K}$. The experimental data (blue trace) is simulated as the $5^{\prime}-\mathrm{dA}-\mathrm{C} 44^{\prime} \cdot$ exchange coupled to $[4 \mathrm{Fe}-4 \mathrm{~S}]^{1+}$ (in a 1:1 ratio, red trace) along with the rest of $[4 \mathrm{Fe}-4 \mathrm{~S}]^{1+}$ (pink trace). 


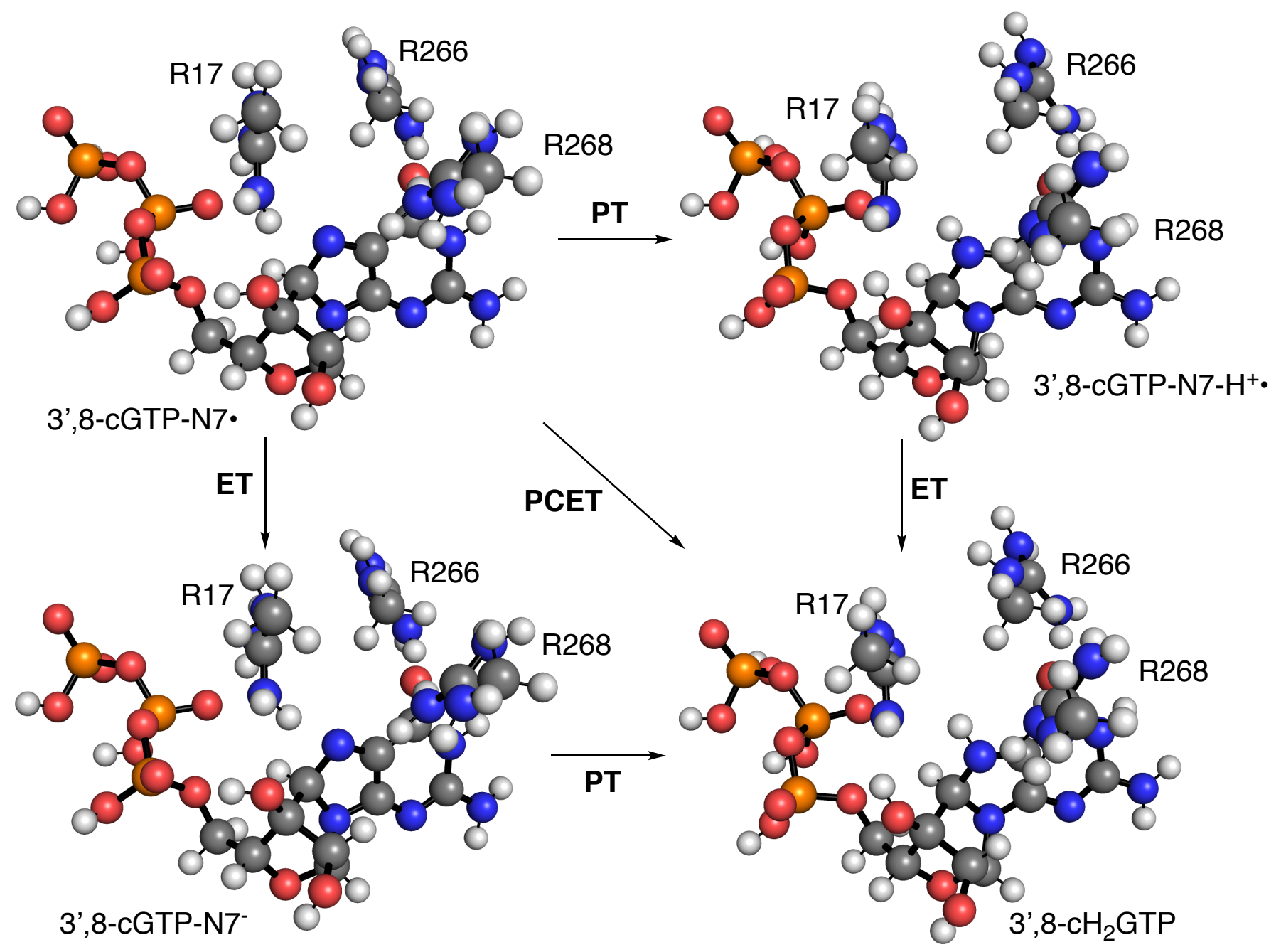

Figure S6. Structures of different states in DFT calculation of aminyl radical reduction.

The 3',8- $\mathrm{CH}_{2} \mathrm{GTP}-\mathrm{N} 7 \cdot$ (top left) is the product state of the previously reported calculation of the transformation of GTP-C3' into 3',8- $\mathrm{CH}_{2}$ GTP-N7•. The structure of 3',8- $\mathrm{CH}_{2} \mathrm{GTP}-\mathrm{N} 7 \cdot$ is calculated based on that of GTP in the crystal structure of MoaA•GTP complex. The positions of triphosphate and O6, N1, and N2 atoms were fixed and ribose was allowed to move. The three catalytically essential residues, R17, R266 and R268, were replaced with methylguanidinium ions. The PT and PCET steps were calculated by a transfer of a proton from R17 to N7 of the intermediate. 


\section{Reference}

1. Maiocco, S. J.; Grove, T. L.; Booker, S. J.; Elliott, S. J., Electrochemical Resolution of the [4Fe-4S]

Centers of the AdoMet Radical Enzyme BtrN: Evidence of Proton Coupling and an Unusual, Low-Potential Auxiliary Cluster. J Am Chem Soc 2015, 137, 8664-8667. 